\title{
Many-Body Interaction Evidenced through Exciton-Trion-Electron Correlated Dynamics
}

\author{
M.T. Portella-Oberli ${ }^{a}$, J.H. Berney ${ }^{a}$, B. Deveaud ${ }^{a}$, \\ V. Ciulin $^{b}$, M. Kutrowski ${ }^{c}$ And T. Wojtowicz ${ }^{c}$ \\ ${ }^{a}$ Institut de Photonique et d'Electronique Quantique, Ecole Polytechnique \\ Fédérale de Lausanne (EPFL), 1015 Lausanne, Switzerland \\ ${ }^{b}$ IQUEST, University of California, Santa Barbara, Ca 93106, USA \\ ${ }^{c}$ Institute of Physics, Polish Academy of Science \\ al. Lotnikow 32/46, 02-668, Warsaw, Poland
}

\begin{abstract}
Interactions among excitons, trions, and electrons are studied in $\mathrm{CdTe}$ modulation-doped quantum wells. These many-body interactions are investigated through the nonlinear dynamical properties in the excitonic complexes using time and spectrally resolved pump and probe techniques. This study is performed as a function of temperature and densities of excitons, trions, and electrons. The results reveal that the nonlinearities induced by trions differ from those induced by excitons and moreover they are mutually correlated. The correlated behavior of excitons and trions manifests itself by crossed trion-exciton effects. We propose that the main source of these correlations is due to the presence of electrons in the quantum well and that its physical origin is the Pauli exclusion-principle. We find that, at $5 \mathrm{~K}$, trions are formed from excitons within $10 \mathrm{ps}$; at $20 \mathrm{~K}$ a thermal equilibrium is reached within 5 ps.
\end{abstract}

PACS numbers: 71.35.Pq, 78.47.+p, 42.65.-k, 78.67.De

\section{Introduction}

Many-body interactions are the main source of nonlinear optical properties in semiconductors. In undoped semiconductors, Coulomb interaction gives rise to the electron-hole bound states (excitons) that play a crucial role in determining the optical properties near the band edge [1]. The nonlinear dynamical properties of excitons are a very direct probe of the many-body interactions occurring 
in quantum wells. The optical spectrum of moderately doped quantum wells also features a trion (charged exciton) resonance, which is situated only a few meV below the exciton line $[2,3]$. The way that many-body interactions are modified by the presence of an additional electron gas is clearly a topic of major interest for applications such as transport of light by a charged exciton [4] and for quantum-information science [5-7]. Another interest of the trion system is that it constitutes the first step in the transition of the optical susceptibility of semiconductor quantum structures from the undoped case to the case of an arbitrary doping level giving the Fermi edge singularity [8, 9].

We present novel results on the dynamical nonlinear optical properties of excitonic complexes in CdTe modulation-doped quantum wells, due to many-body interactions among excitons, trions, and electrons. These were studied through time and spectrally resolved pump-probe experiments. We observe a wealth of phenomena encompassing bleaching, crossed bleaching, induced-absorption, and energy shifts of the resonances. We show that the nonlinearities induced by trions differ from those induced by excitons. Using these differences in the optical nonlinearities of the exciton and trion spectra, we find that, at low temperature, excitons are relaxed to trions in about 10 picoseconds. At higher temperatures, the results reveal that a thermal equilibrium is reached between the exciton and the trion populations. In addition, we find that a correlated behavior of excitons and trions manifests itself by crossed trion-exciton effects. We propose that the main source of these correlations is due to the presence of electrons in the quantum well and that the physical origin of the exciton and trion nonlinear optical effects is the Pauli exclusion-principle. Our results provide a detailed picture of the trion and exciton nonlinear optical dynamics related to many-body scattering process.

\section{Experimental}

The sample studied here has been fully characterized in previous studies [10-13]. It is a one-sided graded modulation-doped CdTe/CdMgTe heterostructure containing a single quantum well of $8 \mathrm{~nm}$. The monolayers of iodine were incorporated in the barrier separated from the quantum well by a spacer of $10 \mathrm{~nm}$. Different amount of iodine donor results, in this way, in different electron concentrations in different parts of the well. The electron concentration can be additionally increased by illuminating the sample with light of energy higher than the energy gap of the barrier. The mechanism of this electron density control is based on the competition between the quantum well and surface states, which can both trap carriers $[14,15]$. In Fig. 1, we plot the linear reflectivity spectra of the less doped part of the sample for several light illumination intensities, resulting in electron concentrations estimated to be between $2 \times 10^{10}$ electrons $/ \mathrm{cm}^{2}$ and $9 \times 10^{10}$ electrons $/ \mathrm{cm}^{2}$. These spectra reveal, as the electron concentration is increased, that: the trion peak gains intensity relative to the exciton peak; the 


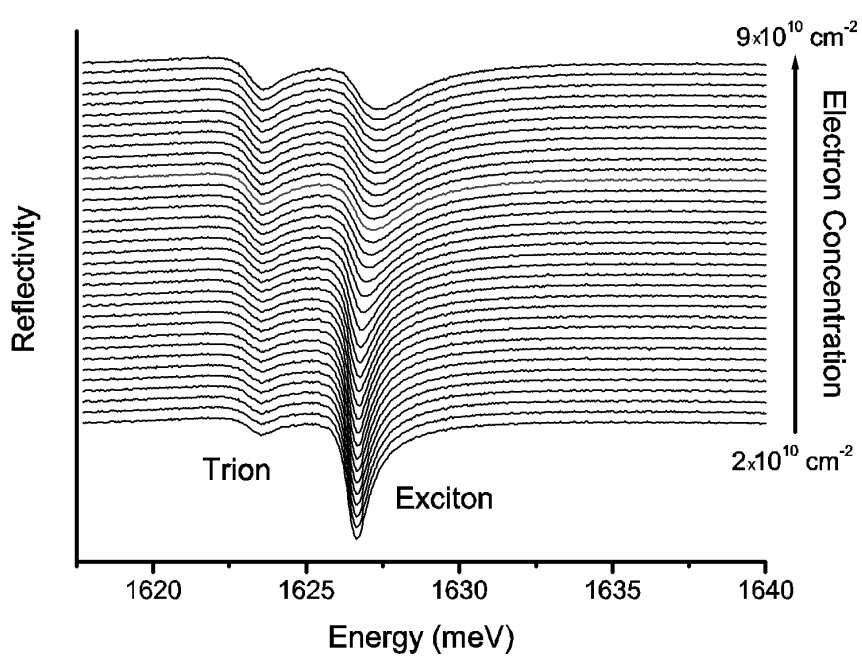

Fig. 1. Linear reflectivity spectra of the less doped part of the sample for several light illumination intensities. The concentration of electrons is varied from $2 \times 10^{10} \mathrm{~cm}^{-2}$ to $9 \times 10^{10} \mathrm{~cm}^{-2}$.

energy difference between trion and exciton resonances increases, mainly because the exciton resonance shifts to higher energies; furthermore, the trion and exciton lineshapes become asymmetric, due to electrons scattering processes, manifested as a high energy tail on their resonances. All these changes with electron density observed in reflectivity are those expected in absorption spectra. Due to the similarity between reflectivity and absorption in our sample, the absorption changes can be directly quantified on the reflectivity spectrum. This is confirmed by a simple calculation, using the transfer matrix formalism [16]. We obtain that a variation of $1 \%$ of trion and exciton oscillator strength $\left(\Delta f_{\mathrm{T}} / f_{\mathrm{T}}\right.$ and $\Delta f_{\mathrm{x}} / f_{\mathrm{x}}$, respectively) gives rise linearly to a variation in the differential reflectivity $\left(\Delta R_{\mathrm{T}} / R_{\mathrm{T}}\right.$ and $\Delta R_{\mathrm{x}} / R_{\mathrm{x}}$, respectively) of precisely $1.2 \%$ and $1.6 \%$.

We have calculated the reflectivity spectra using the transfer matrix model [16]. The shape of the reflectivity spectra strongly depends on the width of the different layers composing the sample. A transfer matrix approach takes into account the precise structure of the sample and yields simulated reflectivity and absorption spectra. The exciton and the trion resonances in the well are introduced by adding two frequency-dependant Lorentzian lines to the constant complex dielectric background [17]. These contributions to the dielectric function should not be properly Lorentzians because electron scatterings broaden and asymmetrize to the lines [18]. Calculations considering electron scattering contributions will be the subject of a forthcoming publication [19]. In Fig. 2, we plot the calculated and measured linear reflectivity spectra of the part of the sample on which we have performed the experiments. The electron concentration is of about 


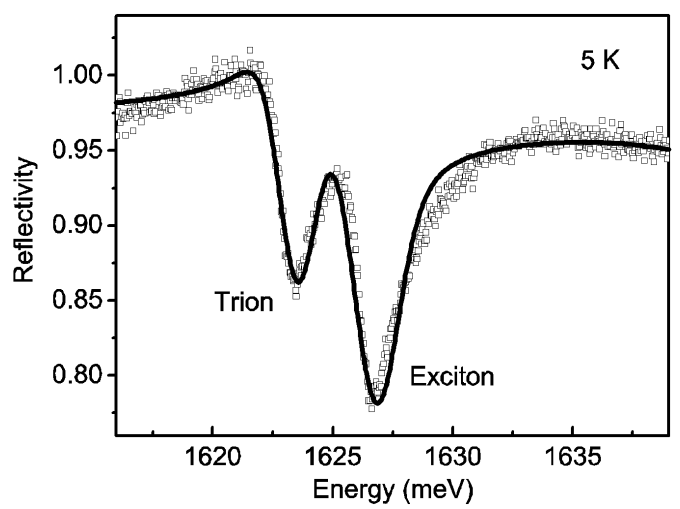

Fig. 2. Calculated (full line) and measured (square symbols) linear reflectivity spectra from a part of the sample with electron concentration of $4.5 \times 10^{10} \mathrm{~cm}^{-2}$, without illumination.

$4.5 \times 10^{10}$ electrons $/ \mathrm{cm}^{-2}$ without illumination of the sample. In our sample the measured and calculated reflectivity spectra have an absorption-like line shape. This is because the quantum well is situated at a distance close to $\lambda / 4$ from the surface (the cap layer thickness is $50 \mathrm{~nm}$ ). The reflectivity spectrum would be dispersive for cap layers with other thicknesses. Our sample has been grown with precisely defined parameters to obtain the desired linear reflectivity spectrum that allows probing absorption features from the reflectivity spectrum.

We use temporally and spectrally resolved pump and probe experiments to investigate the nonlinear behavior of the complex excitonic spectrum [20]. We excite the sample with a narrow tunable 1.3 picosecond pump pulse on resonance with either the trion or the exciton line. The modifications of the optical spectrum are probed by a broad 100 femtosecond probe pulse. The pump-probe nonlinear spectra are detected at different delay times between pump and probe pulses. The differential reflectivity spectrum $\Delta R=\left(R-R_{0}\right) / R_{0}$ is calculated for each delay time, $R_{0}$ and $R$ being the unexcited and excited reflectivity spectra, respectively. The experiments are performed with cross-linearly polarized pulses for several temperatures and densities of excitons and trions.

\section{Resonant excitation of neutral excitons}

In Fig. 3, we plot a set of differential reflectivity spectra, obtained when pumping selectively at the exciton resonance, for several delay times between pump and probe pulses and at temperatures of $5 \mathrm{~K}, 10 \mathrm{~K}$, and $20 \mathrm{~K}$. These differential spectra all show a clear blue-shift of the exciton line. This renormalization of the exciton resonance has been extensively studied in undoped-semiconductor quantum wells [21-24] and, is attributed to short-range fermion exchange [22], which is a repulsive electron-electron and hole-hole interaction. The exciton blue-shift 


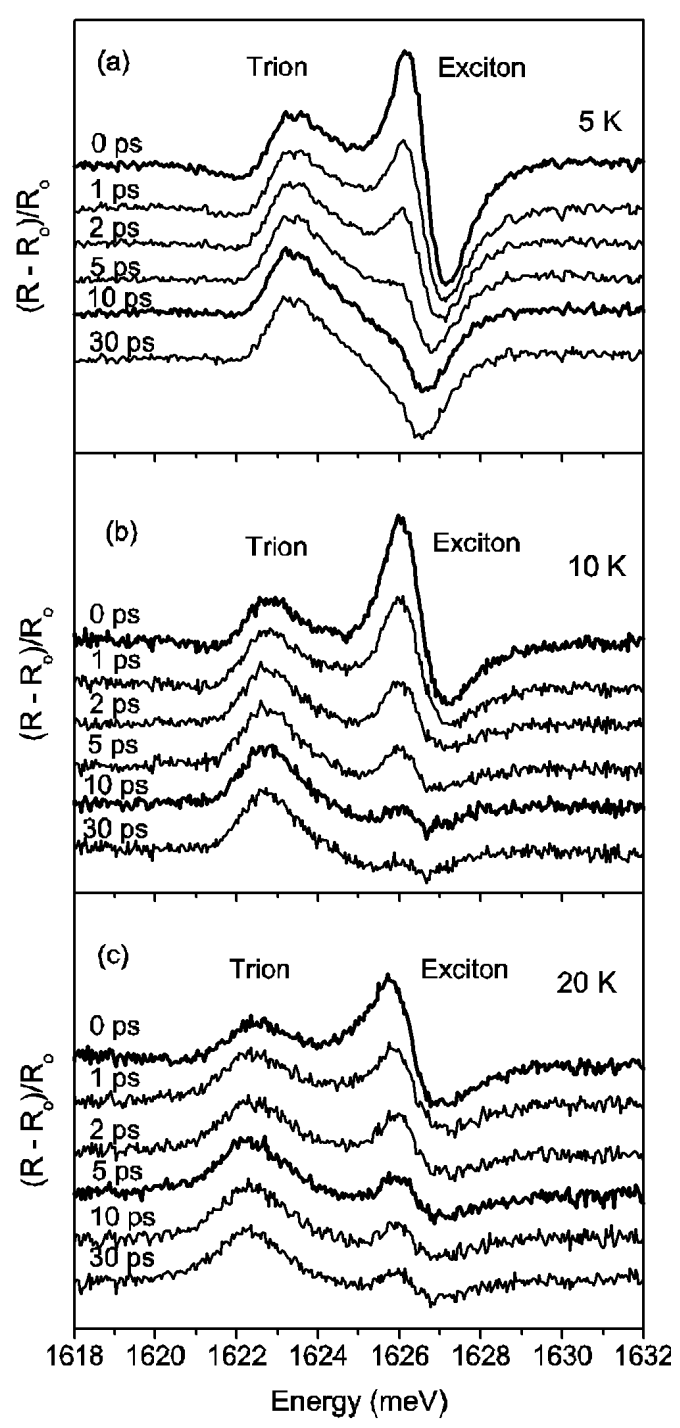

Fig. 3. Differential reflectivity spectra obtained by pumping at exciton resonance, at several delay times between pump and probe pulses and at temperatures of: (a) $5 \mathrm{~K}$, (b) $10 \mathrm{~K}$, and (c) $20 \mathrm{~K}$. The exciton density is $3 \times 10^{10} \mathrm{~cm}^{-2}$. The trion red-shift is observed only at $5 \mathrm{~K}$ and the trion bleaching broadens with temperature.

appears in two-dimensional semiconductors [23] because the long-range Coulomb correlation effect is strongly reduced [22]. The latter corresponds to the van der Waals attraction in the exciton gas and would induce a red-shift on the exciton resonance. Therefore, this existence of the exciton blue-shift, as an exciton population is generated, is the signature that the long-range Coulomb correlation has 
an effect that we can neglect compared to the more efficient short-range fermion exchange interaction, in our sample.

The photo-generated exciton population also induces nonlinear effects on the trion resonance. We observe both a bleaching and a red-shift of the trion resonance, which depend on temperature (Fig. 3) and exciton density [20]. As seen in Fig. 3, the trion red-shift is present only at low temperatures (Fig. 3a) and both crossed effects already occur at zero delay time.

As concerns the time evolution of the nonlinear trion signal, it is important to note (Fig. 3) that the trion bleaching signal does not increase with time, as would be expected if this bleaching was only due to a trion population. The almost constant value of the trion bleaching shows that this bleaching evolves according to two opposite contributions of the same order of magnitude. The contribution due to the trion population which increases with time, and that due to the exciton population which decreases with time. Thus, we attribute the trion bleaching, at short times, to the phase-space filling of the optically-accessible $k$-space by the photo-generated excitons, resulting in a blocking of the trion transition. This result evidences that excitons and trions share the same $k$-space and originate from the same ground state.

The red-shift of the trion line, i.e. an increase in the low energy edge absorption of the trion transition, can only be observed in the first $2.5 \mathrm{ps.} \mathrm{At} \mathrm{short}$ times, there are only electrons and the photo-generated excitons in the quantum well. We suggest that the most likely origin of the red-shift effect is a change in the distribution of electrons with the presence of photo-generated excitons through the exciton-electron interaction.

The exciton-electron interaction is already evidenced in the linear reflectivity spectra when a tail at higher energy of the exciton resonance shows up due to the shake-up process. Moreover, this electron-exciton interaction is an ultrafast process, indeed it has been reported [25] that the exciton dephasing time decreases with the presence of an incoherent electron population in the quantum well. In our experiment, we estimate the exciton dephasing time to be 1 ps or even less $[20,26]$. Thus, as excitons are excited they interact with electrons, which results in an increase of the electron occupation at higher energies favoring the trion transitions at lower energies [20]. The trion red-shift is then observed at short times and, at later times, it is masked by the bleaching effect due to trion formation from the exciton population (see below). We propose that excitons interact with electrons by short-range fermion exchange because the long-range Coulomb interaction is a less important effect, as has been indicated by the blue-shift of the exciton resonance.

At higher temperatures, $10 \mathrm{~K}$ and $20 \mathrm{~K}$, the red-shift of the trion resonance is not observed (Figs. 3b and c). At these temperatures, the homogeneous broadening of the electron distribution is essentially due to electron-acoustic phonon scattering, which is the dominant electron scattering process in this temperature 
range [11]. Therefore, the exciton-electron interaction does not modify the electron distribution enough to induce an increase in the trion absorption at low energies. As a result, at higher temperatures, when excitons are created we only observe a broad bleaching of the trion resonance (Figs. 3b and c). This bleaching signal is the consequence of the phase-space filling mainly by the excitons, at short times and, at longer times, is also attributed to trions while the thermal equilibrium between trion and exciton populations is reached (see below).

\section{Resonant excitation of trions}

The nonlinear optical effects in the reflectivity spectrum for resonantly created trions are notably different from those induced by an exciton population. Figure 4 displays the time evolution of the differential reflectivity spectrum when the sample is excited at the trion resonance, at $5 \mathrm{~K}$. The trion resonance is bleached without any shift in energy and we find an induced absorption of the exciton line, which evolves with the trion bleaching signal.

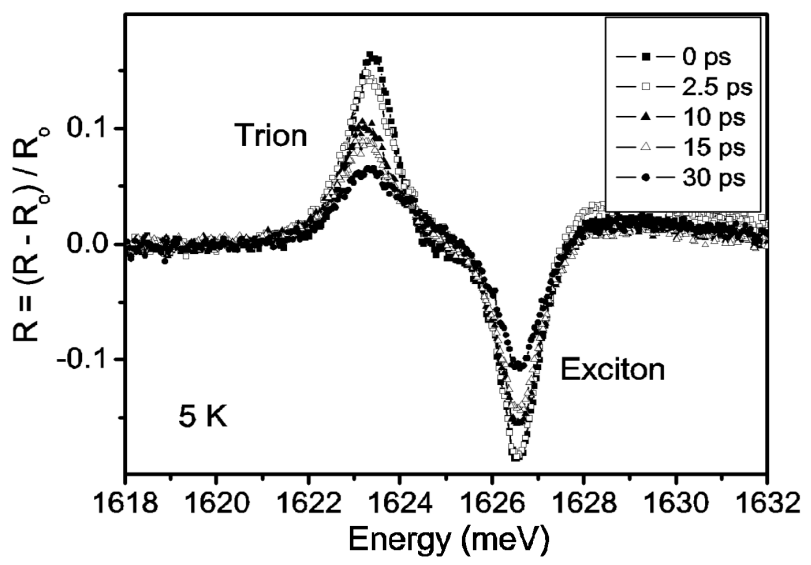

Fig. 4. Differential reflectivity spectra obtained by pumping at trion resonance, at different delay times between pump and probe pulses at $5 \mathrm{~K}$. The trion density is about $2 \times 10^{10} \mathrm{~cm}^{-2}$.

The absence of an energy shift of the trion resonance upon resonant pumping shows the ineffectiveness of the trion-trion interaction. Note that an exciton population induces a blue-shift of the exciton resonance (Fig. 3), which has been attributed to the short-range exciton-exciton exchange interaction. Therefore, we are led to assert that exciton-exciton scattering is more efficient than trion-trion scattering. This is corroborated with the results from four-wave mixing experiment [26], in which we have measured the dephasing time for excitons and trions for the same density, at low temperature and low electron concentration. We have found that excitons dephase much faster than trions. The exciton diphasing 
time $T_{2}$ has been found to be $10 \mathrm{ps}$ and the $T_{2}$ of trions much larger, at $28 \mathrm{ps}$. To our knowledge, there is no theoretical investigation on the short-range exchange between trions, but our results suggest that the trion-trion exchange interactions are less efficient than the exchange interaction between excitons. The localization of trions may play an important role in making the inter-trion exchange process inefficient. Trions have indeed been found to be more localized than excitons, at low temperature [26]. At higher temperatures, trions are no longer localized [26]. We have performed experiments at higher temperatures [20, 27] and we found that there is no shift of the trion resonance. This result shows that trion-trion interaction is always inefficient whether they are localized or not. The trion resonance is broadened due to scattering with acoustic phonons and additionally, the induced absorption of excitons persists as a correlated effect between excitons and trions [27]. These results demonstrate, without ambiguity, that the trion-trion interaction is not the dominant scattering process for trions.

In order to evaluate these trion and exciton oscillator strength variations (Fig. 4), the spectrally integrated bleaching signal of trions and the spectrally integrated induced absorption signal of excitons are divided by 1.2 and by 1.6 (factors calculated with matrix transfer, see above), respectively. In Fig. 5, they are plotted as a function of time for several excitation powers. These results show that: (a) as trions are created in the well, the induced absorption gained by excitons reaches similar amplitude to the bleaching experienced by trions and (b) the induced absorption of excitons decays with trion bleaching. These demonstrate correlated effects acting on both signals.

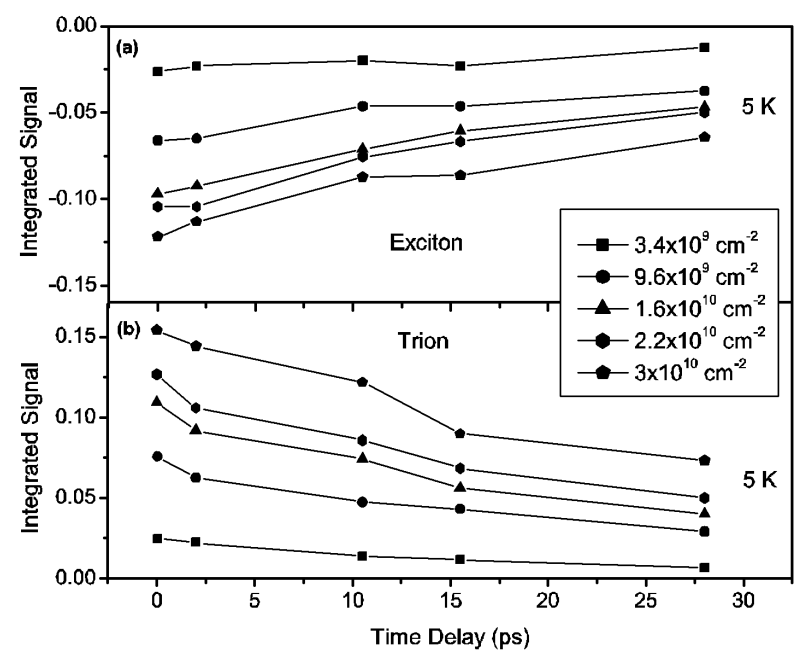

Fig. 5. (a) Spectrally integrated induced absorption signal of excitons and (b) spectrally integrated bleaching signal of trions, as a function of delay time, for several excitation powers at the trion resonance, at $5 \mathrm{~K}$. 
It is known that, in undoped quantum wells, the oscillator strength of excitons is very large and, in modulation-doped quantum wells, the exciton loses its oscillator strength, due to the presence of an electron gas [3]. In our experiment we obtain an increase in the excitonic absorption when the electron population is transformed into a trion population. We attribute the gain of exciton oscillator strength, when trions are created, as resulting from the changes in the screening of excitons via a short-range electron-electron exchange. It is worth noting that the electron-electron exchange interaction produces effects similar to that of the classical Coulomb interaction: the former also produces an "exchange hole", by repelling all the electrons in the same spin state, just like the "correlation hole" in the latter [1]. Therefore, this extra "positive charge" generated from the electron exchange process screens the exciton Coulomb potential. As trions are photo-generated, there are fewer electrons in the electron gas to screen the exciton and the exciton gains back some oscillator strength. As a trion recombines, the electron is released back to the electron gas and is able again to screen excitons. Thus, we observe a recovery of the induced absorption signal of excitons with time (Figs. 4 and 5). This result reveals that electrons interact much more efficiently among themselves than trions do, which suggests that excitons are more dynamically screened by electrons than by trions. The above explanation in terms of more efficient screening of excitons by electrons than by trions confirms the ineffectiveness of the trion-trion interaction and therefore the absence of an energy shift of the trion resonance upon resonant pumping.

We attribute the bleaching of the trion line when a trion population is generated to the phase-space filling. In Fig. 6a we plot the differential reflectivity spectra obtained in pump-probe experiments by excitation at the trion resonance, at zero delay time and for several trion densities. We find that the bleaching of the trion resonance and the induced absorption of the exciton line always have similar amplitudes despite the densities of trion generated in the quantum well. In the inset of Fig. 6b, we plot the cw reflectivity spectra for two electron concentrations in the quantum well. We calculate their differential reflectivity, which is plotted in Fig. 6b: this spectrum reveals the change in the reflectivity as the electron concentration is varied by $1 \times 10^{10} \mathrm{~cm}^{-2}$. In this case, the trion bleaching and the induced absorption of excitons are only due to the decreasing concentration of electrons in the electron gas. We compare this differential spectrum (Fig. 6b) with that recorded in the pump-probe experiment with a similar trion density of about $9.6 \times 10^{9} \mathrm{~cm}^{-2}$. In contrast, we observe that the bleaching of the trion resonance is much larger when a trion population is generated. This difference originates from the phase-space filling of trions. Therefore, the trion bleaching in the pump-probe experiments comes from both the phase-space filling of trions and the phase-space unfilled by electrons. The induced absorption of excitons is already a very large effect as the electron gas concentration is decreased and this effect seems to be less important in the pump-probe signal, when a trion population is present. This 


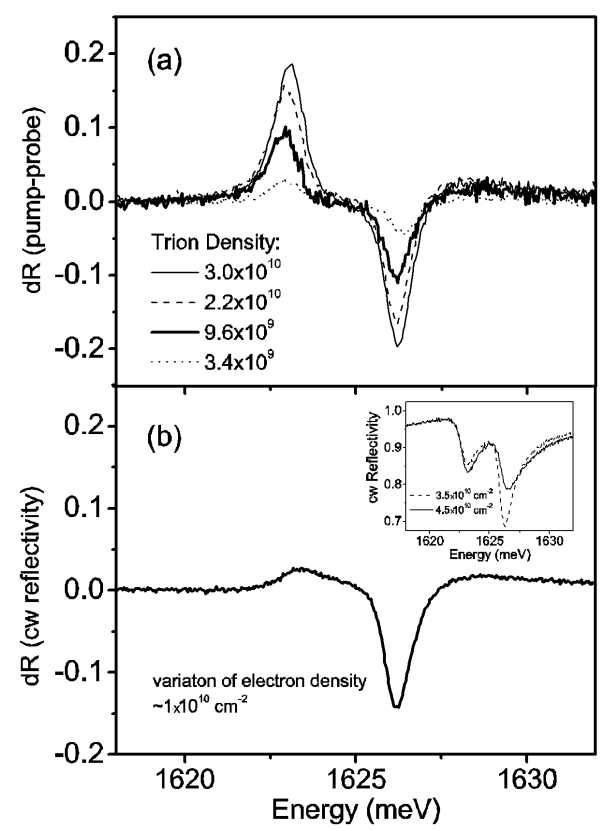

Fig. 6. (a) Differential reflectivity spectra obtained by pumping at trion resonance, at zero delay time between pump and probe pulses and for several trion densities. (b) Differential cw reflectivity spectra obtained from reflectivity spectra are shown in the inset. The variation of electron density between solid and dashed spectra is about $1 \times 10^{10} \mathrm{~cm}^{-2}$. Temperature is $5 \mathrm{~K}$.

difference can be attributed to the phase-space filling by trions: in the same way excitons that have been observed to block the trion transition due to the phase-space filling (above), the presence of trions should block the exciton transition by the same phase-space filling argument. A screening effect on excitons by trions, much less important than that by electrons, cannot be excluded. However, further investigations are needed to get more accurate conclusions about this.

\section{Equilibrium between excitons and trions}

We have shown that the nonlinearities in the optical spectrum of the excitonic complexes induced by the presence of either an exciton or a trion population generated in the quantum well are quite different. We use these very distinct optical nonlinearities in the spectra to investigate the dynamics of trion formation from an exciton population. We generate an exciton population in the quantum well by exciting the sample at the exciton resonance, and we can pursue the process of trion formation from an exciton population, following the time change of the pump-probe signal (Fig. 3). In Fig. 3a we show that excitons are relaxed to trions in about 10 ps at $5 \mathrm{~K}$ : the spectrum at zero delay, due to an exciton population, 
evolves to spectra where trions and excitons cohabit in the well [28] until it is transformed into a spectrum (as in Fig. 4) that is the signature that only trions are present in the quantum well. At higher temperatures, we find that a thermal equilibrium is reached between both populations. This is seen in Fig. $3 \mathrm{~b}$ and c by the stability of the spectra plotted from $10 \mathrm{ps}$ and 5 ps to $30 \mathrm{ps}$, respectively. Therefore, at $10 \mathrm{~K}$ thermal equilibrium is reached within $10 \mathrm{ps}$, and at $20 \mathrm{~K}$ it occurs within 5 ps.

In conclusion, we have studied many-body effects in the presence of electrons, excitons, and trions in CdTe modulation doped quantum wells. We found significant differences between the nonlinear effects induced by an exciton and by a trion population. In both cases, either in the presence of the excitons or trions, the electron gas is perturbed inducing crossed effects in the reflectivity spectrum: A red-shift of trions in the presence of an exciton population due to exciton-electron interaction, and an induced absorption of excitons when trions are present providing evidence for the dynamical screening of excitons by electrons. The poor screening by trions together with the lack of energy renormalization of the trion resonance with trion population suggests that trion-trion interactions are very ineffective. On the other hand, the blue-shift of the exciton line induced by an exciton population shows that the short-range fermion exchange is a much more important interaction than the long-range Coulomb interaction in our sample. Crossed bleaching effects strongly suggest that excitons and trions share phase space. By following the evolution of the nonlinearities of the complex excitonic spectra, we find that trions are formed from excitons within 10 ps at $5 \mathrm{~K}$ and, at higher temperatures, a thermal equilibrium between exciton and trion populations is reached within 5 ps.

We would like to mention that parallel studies have been performed in $p$ -doped quantum wells with selected spin populations of excitons, trions, and electrons, which found effects similar to those we have described here [29].

\section{Acknowledgment}

This work was partially supported by the Swiss National Science Foundation. We are grateful to Cristiano Ciuti, M. Combescot, Daniel Oberli and R. Zimmermann for enlightening discussions.

\section{References}

[1] D.S. Chemla, D.A.B. Miller, J. Opt. Soc. Am. B 2, 1155 (1984).

[2] M.A. Lampert, Phys. Rev. Lett. 1, 450 (1958).

[3] K. Kheng, R.T. Cox, Y. Merle d'Aubigné, F. Bassani, K. Saminadayar, S. Tatarenko, Phys. Rev. Lett. 71, 1752 (1993).

[4] D. Sanvito, F. Pulizzi, A.J. Shields, P.C.M. Christianen, S.N. Holmes, M.Y. Simmons, D.A. Ritchie, J.C. Maan, M. Pepper, Science 294, 837 (2001).

[5] M. Nielsen, I. Chuang, Quantum Computation and Quantum Information, Cambridge University Press, Cambridge 2000. 
[6] C. Piermarocchi, Pochung Chen, L.J. Sham, D.G. Steel, Phys. Rev. Lett. 89, 167402 (2002).

[7] E. Pazy, E. Biolatti, T. Calarco, I. D’Amico, P. Zanardi, F. Rossi, P. Zoller, Europhys. Lett. 62, 175 (2003).

[8] P. Hawrylak, Phys. Rev. B 44, 3821 (1991).

[9] S.A. Brown, J.F. Young, J.A. Brum, P. Hawrylak, Z. Wasilewski, Phys. Rev. B 54, R11082 (1996).

[10] T. Wojtowicz, M. Kutrowski, G. Karczewski, J. Kossut, Appl. Phys. Lett. 73, 1379 (1998).

[11] V. Ciulin, P. Kossacki, S. Haacke, J.-D. Ganière, B. Deveaud, A. Esser, M. Kutrowski, T. Wojtowicz, Phys. Rev. B 62, R16310 (2000).

[12] V. Ciulin, P. Kossacki, S. Haacke, J.-D. Ganière, B. Deveaud, A. Esser, M. Kutrowski, T. Wojtowicz, Phys. Status Solidi B 227, 307 (2001).

[13] M. Kutrowski, Ph.D. Thesis, Institute of Physics PAS, Warsaw 2001 (in Polish).

[14] M. Kutrowski, P. Kossacki, unpublished.

[15] S. Glasberg, H. Shtrikman. I. Bar-Joseph, Phys. Rev. B 63, 113302 (2001).

[16] V.S. Sasvona, L.C. Andreani, P. Schwendimann, A. Quatropani, Solid State Commun. 93, 733 (1995).

[17] L.C. Andreani, G. Panzarini, A. Kavokin, M.R. Vladimirova, Phys. Rev. B 57, 4670 (1998).

[18] G. Ramon, A. Mann, E. Cohen, Phys. Rev. B 67, 045323-1 (2003).

[19] J.H. Berney, M.T. Portella-Oberli, B. Deveaud, to be published.

[20] M.T. Portella-Oberli, V. Ciulin, J.H. Berney, M. Kutrowski, T. Wojtowicz, B. Deveaud, Phys. Rev. B 69, 235311 (2004).

[21] N. Peyghambarian, H.M. Gibbs, J.L. Jewell, A. Antonetti, A. Migus, D. Hulin, A. Mysyrowics, Phys. Rev. Lett. 53, 2433 (1984).

[22] S. Schmitt-Rink, D.S. Chemla, D.A.B. Miller, Phys. Rev. B 32, 6601 (1985).

[23] D. Hulin, A. Mysyrowicz, A. Antonetti, A. Migus, W.T. Masselink, H. Morkoç, H.M. Gibbs, N. Peyghambarian, Phys. Rev. B 33, 4389 (1986).

[24] G. Manzke, Q.Y. Peng, K. Hennenberger, U. Neukirch, K. Wundke, J. Gutowski, D. Hommel, Phys. Rev. Lett. 80, 4943 (1998).

[25] A. Honold, L. Schultheis, J. Kuhl, C.W. Tu, Phys. Rev. B 40, 6442 (1989).

[26] M.T. Portella-Oberli, V. Ciulin, S. Haacke, J.-D. Ganière, P. Kossacki, M. Kutrowski, T. Wojtowicz, B. Deveaud, Phys. Rev. B 66, 155305 (2002).

[27] M.T. Portella-Oberli, V. Ciulin, J.H. Berney, M. Kutrowski, T. Wojtowicz, B. Deveaud, Phys. Status Solidi C 1, 484 (2004).

[28] M.T. Portella-Oberli, V. Ciulin, M. Kutrowski, T. Wojtowicz, B. Deveaud, Phys. Status Solidi B 238, 513 (2003).

[29] P. Plochocka, P. Kossacki, W. Maslana, J. Cibert, S. Tatarenko, C. Radzewicz, J.A. Gaj, Phys. Rev. Lett. 92, 177402-1 (2004). 